\title{
Clinical Study of Risk Factors, Clinical Presentation and Management of Cellulitis Lower Limb
}

\author{
G.R. Balaji Sharma ${ }^{1}$, K. Sathik Mohamed Massodu ${ }^{2}$, A. Collins ${ }^{3}$, G. Parthipan ${ }^{4}$ \\ ${ }^{1}$ Assistant Professor, Department of General Surgery, Tirunelveli Medical College and Hospital, Tamilnadu, ${ }^{2}$ Assistant \\ Professor, Department of General Surgery, Tirunelveli Medical College and Hospital, Tamilnadu, ${ }^{3}$ Post Graduate, Department \\ of General Surgery, Tirunelveli Medical College and Hospital, Tamilnadu, ${ }^{4}$ Post Graduate, Department of General Surgery, \\ Tirunelveli Medical College and Hospital, Tamilnadu, India
}

Corresponding author: Dr. K.Sathik Mohamed Massodu, Assistant Professor, Department of General Surgery, Tirunelveli Medical College and Hospital, Tamilnadu, India

DOI: http://dx.doi.org/10.21276/ijcmsr.2019.4.2.39

How to cite this article: G.R. Balaji Sharma, K. Sathik Mohamed Massodu, A. Collins, G. Parthipan. Clinical study of risk factors, clinical presentation and management of cellulitis lower limb. International Journal of Contemporary Medicine Surgery and Radiology. 2019;4(2):B176-B180.

\section{A B S T R A C T}

Introduction: Cellulitis is defined as inflammation of skin and subcutaneous tissue, characterised by erythema, swelling, warmth and pain. It can affect any part but most commonly lower limb is involved. The identification of risk factors and timely intervention of lower limb cellulitis will reduce the morbidity and mortality. The aim of the study was to assess the risk factors of cellulitis lower limb and to study the clinical presentation and management of cellulitis lower limb.

Material and methods: A prospective study was carried out in 100 Patients with cellulitis of lower limb admitted in general surgery OPD in Tirunelveli Medical College.

Results: Most of the patients affected were elderly population explained by poor immune response and associated comorbidities. Males (85\%) affected more than females. Most patients had unilateral involvement (94\%). High grade cellulitis are present in most cases. Regarding risk factors diabetes mellitus is more common followed by infected traumatic ulcers and post bite cellulitis. Pus culture showed staphylococcus aureus and streptococcus species are most common. Piperacillin tazobactam and imipenem are the most sensitive antibiotics in majority of cases. Doppler performed showed no patients in the study had deep venous thrombosis. Osteolytic changes were noticed in $12 \%$ of population. $13 \%$ of patients were managed conservatively, $75 \%$ of patients required wound debridement and fasciotomy, $12 \%$ required amputation. $61 \%$ of patients had resultant wound which healed by secondary intention, rest were treated by split skin grafting. $1 \%$ population expired because of septicaemia.

Conclusion: This study on cellulitis lower limb found out that diabetes mellitus is the most common risk factor. Early screening and good glycaemic control prevent the incidence of cellulitis lower limb. Hospital admission for severe forms of cellulitis, appropriate and emergency surgical interventions as needed, employing culture directed antibiotics, managing comorbidities can salvage limb and lives.

Keywords: Cellulitis, Lower Limb, Clinical Presentation, Management

\section{INTRODUCTION}

Cellulitis is a superficial, diffuse, spreading infection of the skin. Its overall frequency is approximately 199 per 100,000 person-years, with nearly equal rates in men and women. The incidence increases about 3.7\% for each year of age or about $43.8 \%$ per each 10 -year increment. ${ }^{1}$ Aside from age, a systemic factor associated with lower limb cellulitis is obesity., ${ }^{2,3}$ Local predisposing factors include previous cellulitis in the area, ${ }^{2,4}$ venous insufficiency, ${ }^{3}$ edema (especially lymphedema), ${ }^{3-5}$ and a skin surface disrupted by ulceration, trauma, or dermatologic conditions, such as eczema. ${ }^{2-5}$ The reservoir for bacteria causing cellulitis is often the toe webs, and, accordingly, colonization of these spaces by Staphylococcus aureus and/or b-hemolytic streptococci, tinea pedis, or interdigital intertrigo (fissuring, scaling, and maceration) are also predisposing factors. ${ }^{2-4} \mathrm{~A}$ previous saphenous venectomy increases the likelihood of cellulitis, which is often recurrent.

The systemic features of infection can be prominent in patients with cellulitis and occasionally precede any signs of cutaneous inflammation by many hours. ${ }^{6}$ The mechanism is uncertain but may represent a vigorous systemic immunologic and inflammatory reaction to materials elaborated by streptococci (such as hyaluronidase and streptokinase) that precede apparent inflammation at the skin site. These systemic findings include fever, chills, tachycardia, hypotension, and confusion, often accompanied by a considerable leukocytosis. Many patients, however, are afebrile. In several series, mostly of hospitalized patients, for example, the frequency of fever has been $22 \%$ to $71 \%$, and may be lower among those treated as outpatients. ${ }^{7-10}$ 
The aim of the study was to assess the risk factors of cellulitis lower limb and to study the clinical presentation and management of cellulitis lower limb.

\section{MATERIAL AND METHODS}

This prospective study was conducted in Department of General Surgery, Tirunelveli Medical College Hospital in 100 patients who got admitted for lower limb cellulitis and its complications.

History regarding the presenting illness, pain, reddening of the region, swelling of the local part, any ulcerations, blister/ bleb formation was taken. In the clinical examination, general examination of the patient for the presence of the anemia and jaundice has been studied and the nutritional and hydration status of the patient has been recorded. Thorough examination of the cardiac system, respiratory system done and the findings recorded. Abdominal examination and central nervous system have also examined. Vital parameterspulse rate, blood pressure, respiratory rate and temperature have been recorded for all the patients.

The severity involvement of the limb is graded as per the CREST guidelines for grading of cellulitis. All patients who presented in the early stage of cellulitis have been started on parenteral antibiotics, covering gram positive, gram negative and anaerobic organisms. The antibiotic response is monitored and if the disease improves, the patient is maintained on the same antibiotic for seven days. If the patient shows no response or if there was progress in the disease, change in the antibiotic was practiced awaiting the culture and sensitivity pattern. In case of blistering or bleb formation, there is a potential for spontaneous rupture and in those cases proactive aseptic aspiration with or without deroofing of the blister was done. Tension and swelling at the site of cellulitis can lead on to ulceration and there is loss of large amount of exudate. In most patients, there is loss of protective skin barrier and so the patient is more prone for dehydration steps were taken to correct the hydration status and wound dressing is done so as to avoid the maceration of the skin.

In cases of advanced cellulitis, which is evidenced by devitalized bronzy skin, with underlying pus-pointing, with the suspicion of a subcutaneous abscess, sloughed out and macerated skin are thoroughly debrided, and underlying abscess if any is drained.

Surgical regimen practiced is such that, the surgical incisions involved and also extended beyond the area of devitalized skin until the zone of fresh viable tissue is reached, abscesses if any are drained, fasciotomy was done when there was a threat of compartment syndrome. The wounds were well irrigated and hemostasis attained. Any blood loss encountered during the surgical procedures, was dealt with adequate blood transfusion.

Evaluation of the wound has been repeated on the daily basis and if needed, further debridement has been done. Those patients with arterial ulcers, cellulitis due to snake bites and few patients with trauma showed gangrenous changes at the site and showed bony destruction and hence in those individuals amputation as necessary was done so as to remove the persisting port of infection. Patients with life-threatening necrotizing cellulitis, with the threat of impending sepsis syndrome, and with distal decreased blood flow were taken for amputation at appropriate levels. Of all the 100 patients managed, the outcome of the management has been analysed and recorded. The outcome has been recorded in such a way whether the patient had an uneventful recovery, or the patient had developed tissue loss with the resulting raw area which needed further management, or the patient had a residual deformity or the patient had died because of the disease.

Management of the resultant surgical wound has also been studied. It was done with either delayed primary closure or we allowed the wound to heal by the secondary intention. In cases, where the wound edges are unlikely to get approximated for the healing, and those which resulted in the raw area, split thickness skin grafting has been done.

\section{RESULTS}

Totally 100 such patients were included in the study and the result being analysed. Out of the 100 patients studied 1 belonged to $<20$ years and 21-30 years, 5 belonged to the age group 31-40years, 21 belonged to $41-50$ years, 27 belonged to 51-60 years and 45 were from the age group more than 60 years. Out of 100 patients 85 patients are male and only 15 patients are females indicating males are more commonly affected. Grading of cellulitis taken in this study has been done as per CREST criteria. The study is being conducted in the patients who need hospital admission for cellulitis, it covers principally the patients belonging to grades II, III and IV (graph-1). Out of the 100 patients studied maximum number of individuals i.e., 67 individuals belong to the grade III cellulitis, whereas 24 patients and 9 patients belongs to

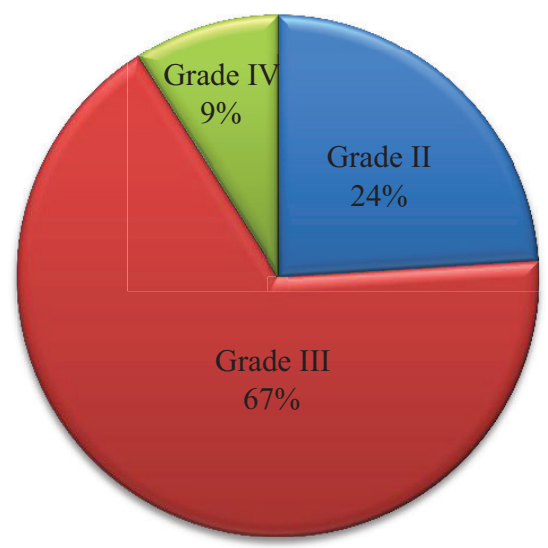

Figure-1: Grade of cellulitis

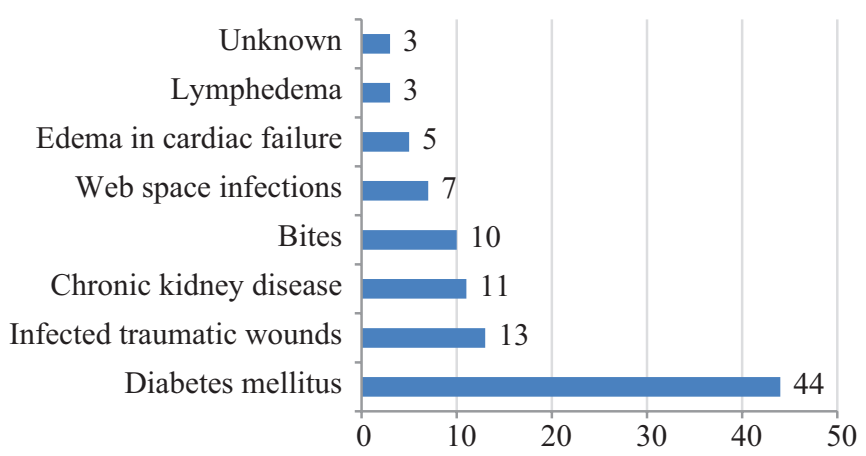

Figure-2: Cause of cellulitis 


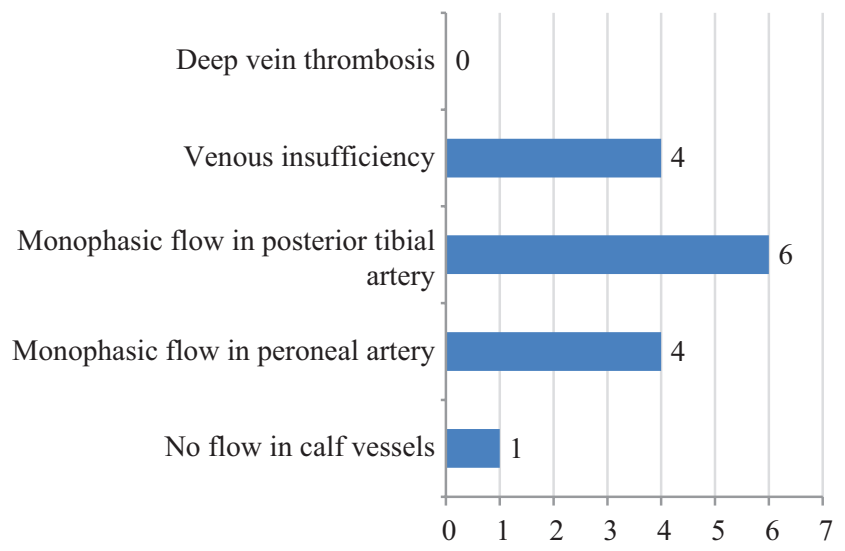

Figure-3: Circulatory changes observed

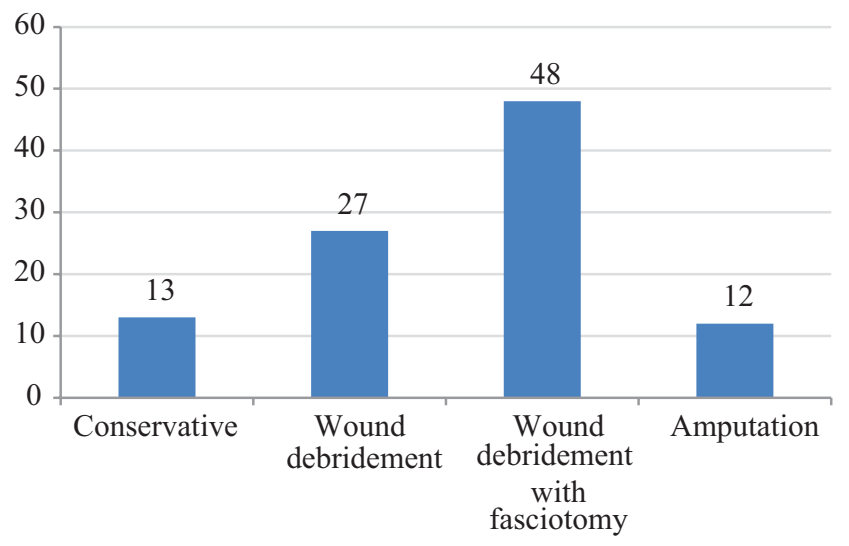

Figure-4: Treatment

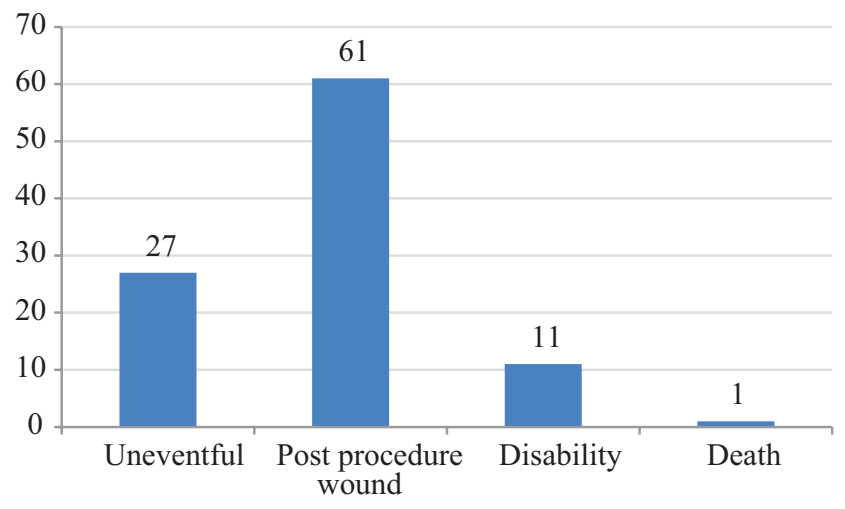

Figure-5: Outcome

grade II and IV respectively. In our study, we have observed that 94 patients had unilateral lower limb involvement and 6 patients had bilateral lower limb involvement, patients with edema going for cellulitis like patients with CKD and cardiac failure, patients with history of barefoot walking with web space infections were candidates who presented with involvement of both lower limbs. Various causes were studied for being responsible for the cellulitis in the study group such as web space infections, diabetes mellitus, bites, infected traumatic ulcers, infected venous ulcers, cellulitis imposing on the lymphedematous limb and in the edematous limb of the renal failure and cardiac failure patients, and in few patients exact cause of the cellulitis could not be made. Diabetes mellitus is responsible for most cases of cellulitis in the study group, followed by the traumatic infected ulcers and post bite cellulitis. It is to be noted that cellulitis superimposing on the lower limb edema occurring in chronic kidney disease, lymphedema and cardiac failure constitutes a considerable proportion as the etiology for the cellulitis in our study group (graph-2). Also in about $3 \%$ individuals the exact cause responsible for the cellulitis is unknown.

Of the 100 patients studied in 47 patients the infection is monomicrobial, in 22 patients the infection is poly-microbial and in about 31 patients no growth has been cultured.

Staphylococcus and streptococcus SP were the predominant organisms responsible for the cellulitis in the study group other organisms responsible include Klebsiella SP, proteus SP, pseudomonas SP, Ecoil.

Among the 100 patients in our study 69 patients were found culture positive. piperacillin - Tazobactam and imipenem are the two group of antibiotics which tend to have the maximum sensitivity for the common organisms causing cellulitis.

Treatment of the individuals varied according to the severity of the disease, some patients were managed conservatively with parenteral antibiotics, the anti-inflammatory agents and limb elevation so as to reduce the associated edema, while majority of the others required surgical wound debridement with or without decompression of the fascial compartment by a fasciotomy. Very few patients needed amputation of the limb. It is observed that around 75 patients in the study group required surgical debridement, 48 of them required decompression of the muscular compartment by means of a fasciotomy. Around $12 \%$ of individuals in the study group required amputation.

The outcome of the treatment done has been studied, whether it is universal, or whether patient remained with a wound that needs, further managements or patient had some residual deformity or the patient had expired because of the comorbidities complicating the disease. All the patients managed conservatively, had an uneventful recovery and among those needed surgical intervention, 61 patients had the residual wound that needed further attention, 11 patients remained with disability computation being done and around 1 patient died because of the disease (graph 3,4).

61 patients in the study group with resultant wound were managed ultimately with either a split thickness or allowed to heal by secondary intention.

\section{DISCUSSION}

Regarding the age distribution, it is evident that as the age increases, the incidence of cellulitis increases, and it has also been studied that the severity of the disease increases with the age, both the inferences correlate, with the literature. This is being explained by the comparatively poorer immune response and the associated comorbidities in the elderly population. There were $85 \%$ males and $15 \%$ females, among the 100 patients and this slightly increased male preponderance is supported by the literature. ${ }^{11}$

Out of the 100 patients studied maximum number of individuals, i.e 67 individuals belong to the grade III cellulitis, whereas 24 patients and 9 patients belong to grade II and IV respectively. In our study, we have observed than $94 \%$ of the patients had unilateral lower limb involvement and 14\% of the patients had bilateral lower limb involvement, but 
according to the literature the incidence of bilateral lower limb involvement is extremely rare. ${ }^{12}$

In our study, cases in whom both lower limb are involved include the patients with edema going for cellulitis like patients with chronic kidney disease and cardiac failure, patients with history of barefoot walking with web space infections, and few patients with unknown etiology. In our study, incidence wise diabetes mellitus is responsible for most cases of cellulitis in the study group, followed by the traumatic ulcers which have been infected and post bite cellulitis. It is to be noted that cellulitis superimposing on the lower limb edema occurring in chronic kidney disease, phedema and cardiac failure constitutes a considerable proportion as the etiology for the cellulitis in our study group. In about 3 individuals the exact cause of cellulitis is unknown. ${ }^{13}$

Of the 100 Patients studied in 47 patients the infection is mono microbial and in 22 patients the infection is poly microbial and in about 31 patients no growth has been cultured. Staphylococcus SP and streptococcus SP were the predominant organisms responsible for the cellulitis in the study group, which correlates with literature. Other organisms observed in the study group include Klebsiella SP, Proteus SP, pseudomonas SP and E coli. ${ }^{14}$

The sensitivity pattern studied for the organisms cultured showed piperacillin tazobactam and imipenem were the two groups of antibiotics which tend to have the maximum sensitivity for the common organisms causing the cellulitis. Cephalosporin group of antibiotics, amikacin, ciprofloxacin and gentamycin are found to be effective in good proportion of individuals. ${ }^{15}$

All the 100 patients in the study group were done Doppler evaluation of the arterial and venous system to study the circulatory change, associated with the cellulitis of the lower limb. We noticed $4 \%$ of the patients showed monophasic flow in peroneal artery and 6\% had monophasic flow in posterior tibial artery and venous insufficiency has been noticed in $4 \%$ of individuals. $1 \%$ of the patients had no flow in the calf vessels and no patients were seen to have deep venous thrombosis. ${ }^{16}$ Bony changes were noticed in the $12 \%$ patients, with cellulitis of the concerned limb in phalanges or the metatarsals in cases of diabetes mellitus. Regarding the treatment, we have noticed that around 75 patients in the study group required surgical debridement, 48 of them required decompression of the some muscular compartment by means of a fasciotomy. $13 \%$ of patients with less severe form of cellulitis were managed conservatively with parenteral antibiotics, the anti-inflammatory agents and limb elevation so as to reduce the associated edema $12 \%$ of individuals in the study group required amputation, because of the loss of almost all viable soft tissues and the possibility of sepsis syndrome because of the badly infected limb. Regarding the outcome of the management, almost all the patients managed conservatively had uneventful recovery, around $61 \%$ of the patients had the residual wound that needed further attention, $11 \%$ of the patients remained with disability (amputation being done), and around 1\% of the patient died because of the comorbidities complicating the illness, especially diabetes mellitus. ${ }^{17}$ In $28 \%$ of the patients, resultant wounds persisted as the raw area after preparing the same, they were managed with split skin grafting, and remaining $72 \%$ of the wounds were allowed to heal by secondary intention (graph-5).

\section{CONCLUSION}

This study on lower limb cellulitis found that diabetes mellitus is the most common cause besides traumatic infected ulcer, post bite cellulitis, chronic kidney disease also contributing. Early diabetes mellitus screening and good glycaemic control prevent the incidence of cellulitis lower limb. Educating the people regarding proper foot care, foot wear usage can prevent cellulitis occurring due to web space infections, cracks in the sole, trivial trauma in the foot. Hospital admission for the severe forms of cellulitis, appropriate and emergency surgical intervention as needed, employing culture directed antibiotics, managing the comorbidities can salvage the limbs and lives.

\section{REFERENCES}

1. McNamara DR, Tleyjeh IM, Berbari EF, Lahr BD, Martinez JW, Mirzoyev SA, et al. Incidence of lowerextremity cellulitis: a population-based study in Olmsted County, Minnesota. Mayo Clin Proc 2007;82(1):81721.

2. Björnsdóttir S, Gottfredsson M, Thórisdóttir AS, Gunnarsson GB, Ríkardsdóttir $\mathrm{H}$, Kristjánsson M, Hilmarsdóttir I. Risk factors for acute cellulitis of the lower limb: a prospective case-control study. Clin Infect Dis $2005 ; 41$ (4):1416-22.

3. Dupuy A, Benchikhi H, Roujeau JC, Bernard P, Vaillant L, Chosidow O, et al. Risk factors for erysipelas of the leg (cellulitis): case control study. BMJ 1999;318 (6):1591-4.

4. Roujeau JC, Sigurgeirsson B, Korting HC, Kerl H, Paul C. Chronic dermatomycoses of the foot as risk factors for acute bacterial cellulitis of the leg: a case-control study. Dermatology 2004;209 (3):301-7.

5. Mokni M, Dupuy A, Denguezli M, Dhaouli R, Bouassida S, Amri M, et al. Risk factors for erysipelas of the leg in Tunisia: a multicenter case-control study. Dermatology 2006;212 (2): 108-12.

6. Eriksson B, Jorup-Ronstr $€$ om C, Karkkonen K, Sj $€$ oblom AC, € Holm SE. Erysipelas: clinical and bacteriologic spectrum and serological aspects. Clin Infect Dis 1996;23 (5):1091-8.

7. Koutkia P, Mylonakis E, Boyce J. Cellulitis: evaluation of possible predisposing factors in hospitalized patients. Diag Microbiol Infect Dis 1999;34 (4):325-7.

8. Chartrier C, Grosshans E. Erysipelas. Int J Dermatol 1990;29 (5): 459-67.

9. Hook EW, Hooton TM, Horton CA, Coyle MB, Ramsey PG, Turck M. Microbiologic evaluation of cutaneous cellulitis in adults. Arch Intern Med 1986;146 (1):295-7.

10. Musher DM, Fainstein V, Young EJ. Treatment of cellulitis with ceforanide. Antimicrob Agents Chemother 1980;17 (5): 254-7.

11. Ellis Simonsen SM, van Orman ER, Hatch BE, et al. Cellulitis incidence in a defined population. Epidemiol Infect. Apr 2006;134(2):293-9.

12. Garg, A., Lavian, J., Lin, G., Sison, C., Oppenheim, 
M., \& Koo, B. Clinical characteristics associated with days to discharge among patients admitted with a primary diagnosis of lower limb cellulitis. Journal of the American Academy of Dermatology, 2017; 76: 626-631.

13. Dupuy A, Benchikhi H, Roujeau JC, et al. Risk factors for erysipelas of the leg (cellulitis): case-control study, BMJ, 1999;318(2):1591-4.

14. Lowy FD. Staphylococcus aureus infections. N Engl J Med. Aug 20 1998;339(8):520-32.

15. Barrett FF, McGehee RF Jr, Finland M. Methicillinresistant Staphylococcus aureus at Boston City Hospital. Bacteriologic and epidemiologic observations. N Engl J Med. 1968;279(9):441-8.

16. Afzal MZ, Saleh MM, Razvi S, Hashmi H, Lampen R. Utility of Lower Extremity Doppler in Patients with Lower Extremity Cellulitis: A Need to Change the Practice? South Med J. 2015;108(7):439-44.

17. Kinlay S. Management of Critical Limb Ischemia. Circ Cardiovasc Interv. 2016;9(2):e001946.

Source of Support: Nil; Conflict of Interest: None

Submitted: 09-05-2019; Accepted: 10-06-2019; Published online: 30-06-2019 